\title{
Equivalence between the Dirac oscillator and a spin-1/2 fermion embedded in a transverse homogeneous magnetic field: movement in a $(2+1)$-dimensional world
}

\author{
Antonio S. de Castro*1] \\ ${ }^{1}$ Universidade Estadual Paulista "Júlio de Mesquita Filho", Departamento de Física e Química, Guaratinguetá, SP, Brasil
}

Received on June 07, 2019; Revised on July 11, 2019; Accepted on August 11, 2019.

\begin{abstract}
It is shown that the Dirac oscillator in a $(2+1)$-dimensional spacetime can be interpreted as a spin- $1 / 2$ fermion embedded in a transverse homogeneous magnetic field.
\end{abstract}

Keywords: Keywords: Dirac oscillator, relativistic planar motion, transverse homogeneous magnetic field.

The planar Dirac oscillator embedded in a transverse uniform magnetic field has emerged in applications to optical models [1]- [3] and graphene [4]- [10]. In a recent work, it has been shown in a simple way that the planar Dirac oscillator and a spin-1/2 fermion embedded a transverse homogeneous magnetic field are equivalent problems [11]. This achievement, put into effect with $4 \times 4$ realizations of the Dirac matrices in a $(3+1)$-dimensional world, fills with suspicions of wrongdoing many works diffused in the literature. In the present work, even with a content easy to deal with by graduate students in Physics, the same achievement is reached by using $2 \times 2$ realizations of the Dirac matrices in a $(2+1)$-dimensional world.

In the Minkowski spacetime, the behaviour of a spin$1 / 2$ fermion of mass $m$ subject to the most generic set of interactions is governed by the Dirac equation (in natural units $\hbar=c=1$ )

$$
\left(\gamma^{\mu} p_{\mu}-m-\mathcal{V}\right) \Psi=0
$$

where $p_{\mu}=i \partial_{\mu}$ and $\mathcal{V}$ is the interaction potential matrix. The gamma matrices satisfy the algebra $\left\{\gamma^{\mu}, \gamma^{\nu}\right\}=2 g^{\mu \nu}$, where $g^{\mu \nu}$ stands for the matrix element of the Minkowski metric tensor with $g^{00}=-g^{i i}=1, g^{\mu \nu}=0$ if $\mu \neq \nu$, i.e. with signature $(+---)$. With the adjoint spinor $\bar{\Psi}=\Psi^{\dagger} \gamma^{0}$, the requirement $\left(\gamma^{0} \gamma^{\mu}\right)^{\dagger}=\gamma^{0} \gamma^{\mu}$ compels the current density $J^{\mu}=\bar{\Psi} \gamma^{\mu} \Psi$ to obey the continuity equation

$$
\partial_{\mu} J^{\mu}=i \bar{\Psi}\left(\gamma^{0} \mathcal{V}^{\dagger} \gamma^{0}-\mathcal{V}\right) \Psi
$$

in such a way that $J^{\mu}$ is conserved only if $\left(\gamma^{0} \mathcal{V}\right)^{\dagger}=\gamma^{0} \mathcal{V}$ [12]. In this way, the most general Dirac equation can also be written in the form

*Correspondence email address: antonio.castro@unesp.br

$$
i \frac{\partial \Psi}{\partial t}=H \Psi=\left(\gamma^{0} \vec{\gamma} \cdot \vec{p}+\gamma^{0} m+\gamma^{0} \mathcal{V}\right) \Psi
$$

with $H^{\dagger}=H$

In $(3+1)$ dimensions, $\gamma^{\mu}$ can be represented by a $4 \times 4$ matrix and the general interaction potential matrix $\mathcal{V}$ can be written as a linear combination of sixteen $\Gamma$-matrices. Usually, the basis elements are chosen in such a way that the bilinear form $\bar{\Psi} \Gamma \Psi$ has a definite transformation property under Lorentz transformations, as listed in Table 1 (see, e.g. [13]- [14]).

The magnetic field $(\vec{B}=\vec{\nabla} \times \vec{A})$ coupled by the minimal prescription $\vec{p} \rightarrow \vec{p}-q \vec{A}$, for example, corresponds to an interaction potential matrix given by

$$
\begin{aligned}
& \mathcal{V}=-q \vec{\gamma} \cdot \vec{A} \quad \text { (potential matrix for the space } \\
& \text { component of a vector coupling). }
\end{aligned}
$$

On the other hand, the Dirac oscillator is obtained by the nonminimal prescription $\vec{p} \rightarrow \vec{p}-i m \omega \gamma^{0} \vec{r}$, corresponding to

$$
\begin{aligned}
& \mathcal{V}=i m \omega \gamma^{0} \vec{\gamma} \cdot \vec{r} \quad \text { (potential matrix for the } \\
& \text { Dirac oscillator). }
\end{aligned}
$$

Table 1: Basis elements of the $4 \times 4$ matrix space and the Lorentz property of $\bar{\Psi} \Gamma \Psi . I_{4 \times 4}$ is the $4 \times 4$ unit matrix, $\gamma^{5}=i \gamma^{0} \gamma^{1} \gamma^{2} \gamma^{3}$ and $\sigma^{\mu \nu}=i\left[\gamma^{\mu}, \gamma^{\nu}\right] / 2$.

\begin{tabular}{ccc}
\hline$\Gamma$ & number of elements & Lorentz property of $\bar{\Psi} \Gamma \Psi$ \\
\hline$I_{4 \times 4}$ & 1 & scalar \\
$\gamma^{5}$ & 1 & pseudoscalar \\
$\gamma^{\mu}$ & 4 & vector \\
$\gamma^{5} \gamma^{\mu}$ & 4 & pseudovector \\
$\sigma^{\mu \nu}$ & 6 & tensor \\
\hline
\end{tabular}


In $(2+1)$ and $(1+1)$ dimensions, though, $\gamma^{\mu}$ can be represented by a $2 \times 2$ matrix and four matrices form the base of the vector space of all Hermitian $2 \times 2$ matrices. In terms of the unit matrix plus the Pauli matrices $\sigma_{1}$, $\sigma_{2}$ and $\sigma_{3}$, one finds scalar and vector structures in $2+1$ dimensions (in the sense of proper Lorentz transformations), and scalar, pseudoscalar and vector structures in $(1+1)$ dimensions.

The interaction potential matrix of the Dirac oscillator under Lorentz transformations changes depending on the dimensions of the Minkowski spacetime. As a matter of fact, the prescription leading to the Dirac oscillator results in a kind of tensor interaction in $(3+1)$ dimensions that can be interpreted as that one describing the behaviour of a particle with anomalous magnetic moment 15]. On the other hand, the Dirac oscillator exhibits a pseudoscalar Lorentz nature in $1+1$ dimensions [16]. Therefore, it is natural to ask about the Lorentz nature of the interaction potential matrix corresponding to the $(2+1)$-dimensional version of the Dirac oscillator. In order to answer this question, for the sake of generality, we go in reverse. As one might guess from the comments on the previous paragraph, the $(2+1)$-dimensional Dirac oscillator is coupled necessarily by a linear combination of scalar and vector couplings. Choosing the representation $\gamma^{0}=\sigma_{3}$ and $\vec{\gamma}=\sigma_{3} \vec{\sigma}$, with $\vec{\sigma}=\left(\sigma_{1}, s \sigma_{2}\right)$ and $s= \pm 1$, the most general Hamiltonian in $(2+1)$ dimensions can be written as

$$
H=\vec{\sigma} \cdot(\vec{p}-\vec{V})+V^{0}+\sigma_{3}(m+S),
$$

where $V^{\mu}=\left(V^{0}, \vec{V}\right)$, with time component $V^{0}$ and space component $\vec{V}=\left(V^{1}, V^{2}\right)$, denotes the vector potential, and $S$ denotes the scalar potential. Therefore, the most general interaction potential matrix $\mathcal{V}=\gamma^{\mu} V_{\mu}+S$ can be written as

$$
\mathcal{V}=-\sigma_{3} \vec{\sigma} \cdot \vec{V}+\sigma_{3} V^{0}+S
$$

Using the identity

$$
\sigma_{i} \sigma_{j}=\delta_{i j} I_{2 \times 2}+i \sum_{k=1}^{3} \varepsilon_{i j k} \sigma_{k},
$$

where $I_{2 \times 2}$ is the $2 \times 2$ unit matrix, $\delta_{i j}$ is the Kronecker delta and $\varepsilon_{i j k}$ is the Levi-Civita symbol, one can write $\vec{\sigma} \cdot \vec{V}=i \vec{\sigma} \cdot \sigma_{3} \vec{v}$, where $\vec{v}=\left(V^{2},-V^{1}\right)$, in such a way that one ends up with

$$
H=\vec{\sigma} \cdot\left(\vec{p}-i s \sigma_{3} \vec{v}\right)+V^{0}+\sigma_{3}(m+S) .
$$

Note that the interaction potential matrix related to the space component of a vector potential $\vec{V}$ in $(6)$ has been replaced in $(9)$ by that one with the same matrix structure of the Dirac oscillator but with a more general potential function.
We now turn to the the case of a minimally coupled magnetic field $\vec{V}=q \vec{A}$. In particular, for a particle immersed in a uniform magnetic field $\vec{B}=B \widehat{k}$ with $\vec{A}$ expressed in the symmetric gauge $\vec{A}=\vec{B} \times \vec{r} / 2$, one finds

$$
H=\vec{\sigma} \cdot\left(\vec{p}-i s \frac{q B}{2} \sigma_{3} \vec{r}\right)+\sigma_{3} m,
$$

which represents exactly the Dirac oscillator with frequency equal to $\omega=|q| B /(2 m)$.

It has been shown that the interaction potential matrix typical of the Dirac oscillator, i.e. $i \gamma^{0} \vec{\gamma} \cdot \vec{v}$, is precisely that one characteristic of the vector coupling $-\vec{\gamma} \cdot \vec{V}$ in a $(2+1)$-Minkowski spacetime. Then, we showed that the Dirac oscillator for an electrically charged particle can be interpreted as the problem describing a spin- $1 / 2$ fermion minimally coupled to a transverse homogeneous magnetic field.

\section{Acknowledgement}

This work was supported in part by means of funds provided by CNPq (grant 304743/2015-1).

\section{References}

[1] B.P. Mandal and S. Verma, Phys. Lett. A 374, 1021 (2010).

[2] B.P. Mandal and S.K. Rai, Phys. Lett. A 376, 2467 (2012).

[3] Y.L. Hou, Q. Wang, Z.W. Long and J. Jing, Int. J. Theor. Phys. 54, 1506 (2015).

[4] C. Quimbay and P. Strange, arXiv:1311.2021 (2013).

[5] C. Quimbay and P. Strange, arXiv:1312.5251 (2013).

[6] M.J. Bueno, J.L. de Melo, C. Furtado and A.M.M. Carvalho, Eur. Phys. J. Plus 129, 201 (2014).

[7] A. Boumali, Phys. Scr. 90, 045702 (2015).

[8] J. Amaro Neto, M.J. Bueno and C. Furtado, Ann. Physics 373, 273 (2016).

[9] A. Belouad, A. Jellal and Y. Zahidi, Phys. Lett. A 380, $773(2016)$

[10] N. Hatami and M.R. Setare, Phys. Lett. A 380, 3469 (2016).

[11] A.S. de Castro, Rev. Bras. Ens. Fis. 41, e20190094 (2019).

[12] L.B. Castro, Phys. Lett. A 375, 2510 (2011).

[13] J.D. Bjorken and S.D. Drell, Relativistic Quantum Mechanics (McGraw-Hill, New York, 1964).

[14] W. Greiner, Relativistic Quantum Mechanics: Wave Equations (Springer-Verlag, Berlin, 1990).

[15] M. Moreno and A. Zentella, J. Phys. A 22, L821 (1989).

[16] A.S. de Castro and W.G. Pereira, Phys. Lett. A 308, $131(2003)$. 